\title{
BRIEF REVIEW ON SEMILEPTONIC B-DECAYS
}

\author{
GIULIA RICCIARDI \\ Dipartimento di Scienze Fisiche, Università di Napoli Federico II \\ Complesso Universitario di Monte Sant'Angelo, Via Cintia, I-80126 Napoli, Italy \\ and \\ INFN, Sezione di Napoli \\ Complesso Universitario di Monte Sant'Angelo, Via Cintia, I-80126 Napoli, Italy \\ giulia.ricciardi@na.infn.it
}

We concisely review semileptonic B decays, focusing on recent progress on both theoretical and experimental sides.

\section{Introduction}

A precise knowledge of semi-leptonic decays of B mesons brings several advantages to flavour physics. They can be studied in the context of the heavy flavour effective theory. Leptonic and hadronic contributions factorize, and we have a better control of the effects of strong interactions, compared to non-leptonic decays. On the experimental side, they are not helicity suppressed as leptonic decays.

Semileptonic decays contribute considerably to the analysis of the unitarity triangle. The so-called unitarity clock, the circle around the origin in the $\bar{\rho}-\bar{\eta}$ plane [1, is proportional to the ratio $\left|V_{u b} / V_{c b}\right|$, which is most cleanly determined from semileptonic decays. The parameters $\left|V_{c b}\right|$ normalize the whole unitarity triangle. A clean determination of $\left|V_{c b}\right|$ and $\left|V_{u b}\right|$ from tree level processes, that are generally assumed not significantly affected by new physics at the current level of precision, is a valuable input for other new physics sensitive estimates.

The inclusive and exclusive determinations of $\left|V_{c b}\right|$ rely on different theoretical calculations, each with different (independent) uncertainties. The former employs a parton-level calculation of the decay rate as a function of the strong coupling constant and inverse powers of the b-quark mass $m_{b}$. The latter hinges on a parameterization of the decay form factors using heavy quark symmetry and a non-perturbative calculation of its normalization. The inclusive and exclusive experimental measurements make use of different techniques and have, to a large extent, uncorrelated statistical and systematic uncertainties. This independence makes the comparison of $\left|V_{c b}\right|$ from inclusive and exclusive decays a powerful test of our understanding of semileptonic decays. The values of $\left|V_{u b}\right|$ extracted from exclusive and inclusive decays differ by about two standard deviation, and a lot of effort, from both experimental and theoretical side, has been devoted to solve this long-standing puzzle. In this short review we discuss semileptonic $B$ decays, focussing on features where 
recent progress has been reported

\section{Exclusive $\left|V_{c b}\right|$ Extraction}

To extract $\left|V_{c b}\right|$ we analyze the tree level driven $\bar{B} \rightarrow D^{(*)} l \bar{\nu}$ weak decays. The starting point are their differential ratios, needed for taking into account experimental cuts. They can be parameterized in terms of $\mathcal{G}(\omega)$ and $\mathcal{F}(\omega)$ according to the formulas

$$
\begin{aligned}
\frac{d \Gamma}{d \omega}(\bar{B} \rightarrow D l \bar{\nu}) & =\frac{G_{F}^{2}}{48 \pi^{3}}\left(m_{B}+m_{D}\right)^{2} m_{D}^{3}\left(\omega^{2}-1\right)^{3 / 2}\left|V_{c b}\right|^{2}(\mathcal{G}(\omega))^{2} \\
\frac{d \Gamma}{d \omega}\left(\bar{B} \rightarrow D^{*} l \bar{\nu}\right) & =\frac{G_{F}^{2}}{48 \pi^{3}}\left(m_{B}-m_{D^{*}}\right)^{2} m_{D^{*}}^{3}\left(\omega^{2}-1\right)^{1 / 2}\left|V_{c b}\right|^{2} \chi(\omega)(\mathcal{F}(\omega))^{2}(1)
\end{aligned}
$$

neglecting the charged lepton and neutrino masses. $\mathcal{G}(\omega)$ and $\mathcal{F}(\omega)$ are a linear combination of the form factors which parameterize the matrix elements $<D\left(v_{D}\right)\left|V^{\mu}\right| \bar{B}\left(v_{B}\right)>$ and $<D^{*}\left(v_{D}^{*}, \alpha\right)\left|A^{\mu}\right| \bar{B}\left(v_{B}\right)>$, respectively ( $\alpha$ refers to the $D^{*}$ polarization). $\chi(\omega)$ is a known phase space factor. The form factors depend on $\omega=v_{B} \cdot v_{D^{(*)}}$, the product of the heavy quark velocities $v_{B}=p_{B} / m_{B}$ and $v_{D^{(*)}}=p_{D^{(*)}} / m_{D^{(*)}}$. In the $\mathrm{B}$ rest frame, $\omega$ corresponds to the energy of $D^{(*)}$ normalized by its mass, that is to $\omega=E_{D^{(*)}} / m_{D^{(*)}}$. The values of $\omega$ are constrained by kinematics: $w \geq 1$, with largest value $w \simeq 1.5$.

The experiments allow to fit only the product of the form factors multiplied by the CKM parameter, that is $\left|V_{c b} \mathcal{G}(\omega)\right|$ or $\left|V_{c b} \mathcal{F}(\omega)\right|$. Due to the kinematic suppression factors, $\left(\omega^{2}-1\right)^{3 / 2}$ and $\left(\omega^{2}-1\right)^{1 / 2}$, data are taken at $\omega \neq 1$. The results are then extrapolated to $\omega=1$, the so-called nonrecoil point, where $v_{B}=v_{D^{(*)}}$, with leptons back to back and the mesons at rest. At the nonrecoil point, heavy quark symmetries play a useful role in constraining the form factors; with the additional input of $|\mathcal{G}(1)|$ and $|\mathcal{F}(1)|$ coming from theory, a value for $\left|V_{c b}\right|$ can be obtained. The main theoretical problem is the non perturbative evaluation of the operator matrix elements. Lattice and QCD sum rules are the two more common routes toward such evaluation.

Lattice determinations for $\bar{B} \rightarrow D^{(*)} l \bar{\nu}$ decays are generally more precise than in most leptonic and semileptonic decays. Form factors can be described to high accuracy by a normalization and a slope. It is possible to connect $\left|V_{c b}\right|$ with the semileptonic form factors via ratios (or double ratios) where most uncertainties cancel in the heavy-quark symmetry limit. Such ratios tend to reduce most of the normalization uncertainty in the lattice currents, as well as highly correlated statistical errors in the numerator and denominator.

The most recent unquenched lattice calculation for $\bar{B} \rightarrow D l \bar{\nu}$ adopts a $2+1$ dynamical gauge configurations generated by the MILC collaboration, and an action with the Fermilab interpretation. After correcting by a factor of 1.007 for QED, it

a $D$ semileptonic decays are a counterpart of the $B$ ones in the charm sector: for a very recent review see e.g. 2] 
gives 3

$$
\mathcal{G}(1)=1.074 \pm 0.024
$$

The latest more precise data have come in 2008 and 2009 from Babar [4,5]. Using the latest HFAG average [6], which also includes older Aleph, CLEO and Belle measurements, the fit results in

$$
\left|V_{c b}\right| \mathcal{G}(1)=(42.64 \pm 1.53) \times 10^{-3}
$$

which can be turned into the following estimate:

$$
\left|V_{c b}\right|=\left(39.70 \pm 1.42_{\exp } \pm 0.89_{\mathrm{th}}\right) \times 10^{-3}
$$

With respect to the previous HFAG determination, the theoretical error has been slightly reduced, increasing the modest dominance of the experimental uncertainty.

An alternative lattice approach consists in calculating the form factor normalization directly at values $\omega>1$, that may allow more precise determinations, avoiding the large extrapolation to $\omega=1$ and reducing model dependence. It is currently available only in the quenched approximation, that neglects $u, d$, and $s$ quark loops [7,8. This approach, by using 2009 Babar data [5], gives the estimate

$$
\left|V_{c b}\right|=\left(41.6 \pm 1.8 \pm 1.4 \pm 0.7_{F F}\right) \times 10^{-3}
$$

a slightly higher value than (4). The errors are respectively statistical, systematic and due to the theoretical uncertainty in the form factor $\mathcal{G}(1)$.

Measuring the differential rate for $\bar{B} \rightarrow D^{*} l \bar{\nu}$ is easier than for $\bar{B} \rightarrow D l \bar{\nu}$ since the rate is higher and there is no background from mis-reconstructed $\bar{B} \rightarrow D^{*} l \bar{\nu}$. The most recent HFAG experimental fit [6] gives

$$
\left|V_{c b}\right||\mathcal{F}(1)|=(35.90 \pm 0.45) \times 10^{-3}
$$

while one can obtain 9.10

$$
\mathcal{F}(1)=0.908 \pm 0.017
$$

by using lattice with the Fermilab action for $b-$ and $c$-quarks, the asqtad staggered action for light valence quarks, and the MILC ensembles for gluons and light quarks. It includes the enhancement factor 1.007, due to the electroweak corrections to the four-fermion operator mediating the semileptonic decay. This is the latest lattice result and updates, one year later, the first $(2+1)$-flavor lattice calculation of $\mathcal{F}(1) 9$ 11, reducing the total uncertainty on $\mathcal{F}(1)$ from $2.6 \%$ to $1.7 \%$. No lattice calculation is still available at $\omega \neq 1$ for $\bar{B} \rightarrow D^{*} l \bar{\nu}$ decays.

By taking Eq. (6) and Eq. (7), the latest value of $\left|V_{c b}\right|$ from exclusive $B \rightarrow D^{*} l \nu$ reads 6

$$
\left|V_{c b}\right|=\left(39.54 \pm 0.50_{\exp } \pm 0.74_{\mathrm{th}}\right) \times 10^{-3}
$$

where the errors come from experiment and QCD lattice calculation, respectively. This number is in excellent agreement with the result (4) coming from $B \rightarrow D l \nu$ decay. 
Non-lattice results generally give one or two sigma lower form factors, and thus a larger value of $\left|V_{c b}\right|$ exclusive. The $\mathcal{F}(1)$ form factor has recently been calculated using zero recoil sum rules, yielding to

$$
\mathcal{F}(1)=0.86 \pm 0.02
$$

including full $\alpha_{s}$ and up to $1 / m_{5}^{2} 12$ 13. Let us remark that in order to compare the value in Eq. (9) with the lattice result Eq. (7), one has to remove the electroweak factor 1.007 from the latter. By using Eq. (6) in combination with Eq. (9), one obtains

$$
\left|V_{c b}\right|=\left(41.6 \pm 0.6_{\exp } \pm 1.9_{\mathrm{th}}\right) \times 10^{-3}
$$

The precision of non-lattice calculations are affected by deviations from the heavy mass limit, which may impact also lattice calculations, as discussed e.g in Refs. 13, 14. The deviations are estimated to be parametrically larger in the case of $\bar{B} \rightarrow D$ with respect to $\bar{B} \rightarrow D^{*}$. In order to get a more precise prediction for the form factor $\mathcal{G}(1)$, it has been suggested 15 to combine the heavy quark expansion with a "BPS" expansion. The latter is an expansion in the limit where the kinetic energy $\mu_{\pi}^{2}$ is equal to the chromomagnetic moment $\mu_{G}^{2}$ : in this limit

$$
\mathcal{G}(1)=1.04 \pm 0.02
$$

With such estimate one finds 16

$$
\left|V_{c b}\right|=\left(40.7 \pm 1.5_{\exp } \pm 0.8_{\text {th }}\right) \times 10^{-3}
$$

The larger experimental uncertainty with respect to Eq. (10) reflects the above mentioned experimental difficulty in measuring the differential rate for $\bar{B} \rightarrow D l \bar{\nu}$.

\section{3. $B$-Mesons Decays to Excited $D$-Meson States}

Let $\mathbf{J}$ be the total angular momentum of an heavy meson. In the limit where the heavy quark mass is infinity, the spin $\mathbf{s}$ of the heavy quark is conserved and decouples from the total angular momentum of the light degrees of freedom $\mathbf{j}_{\mathbf{1}} \equiv \mathbf{J}-\mathbf{s}$. By definition, $\mathbf{j}_{\mathbf{1}}$ becomes a conserved quantity as well, and its eigenvalue $j_{l}$ a good quantum number, despite the fact that the light-degree of freedom consists of a superposition of the light quark, sea quarks and gluons. In the non-relativistic constituent quark model, the open charm system can be classified as $n^{2 S+1} L_{J}$, where $n$ is the radial quantum number, $L$ corresponds to the eigenvalue of the relative angular momentum between the c-quark and the light degrees of freedom, and $S \equiv s \otimes s_{l}$, $s_{l}$ being the spin of the light valence quark and $s$ the eigenvalue of the heavy quark spin. The experimentally observed charmed mesons are associated with the $1 S, 2 S$, $1 P$, and $1 D$ states of the meson wave function.

The $D$ and $D^{*}$ states correspond to the $1 S$ wave function; they form the multiplet $J^{P}=\left(0^{-}, 1^{-}\right)$, respectively, as given by $J=j_{l} \otimes s=0 \oplus 1$, with $s=j_{l}=1 / 2$.

When $L>0$, states are said orbitally excited. The first orbitally excited bound state correspond to the $1 P$ wave function of the charmed system $(L=1)$. Since 
$j_{l}=L \otimes s_{l}$, it is characterized by $j_{l}=1 / 2$ and $j_{l}=3 / 2$. The former gives the doublet with $J=j_{l} \otimes s=1 / 2 \otimes 1 / 2=0 \oplus 1$, that is $J^{P}=\left(0^{+}, 1^{+}\right)$, denoted as $D_{0}^{*}$ and $D_{1}^{\prime}$, respectively. The latter gives the doublet with $J=j_{l} \otimes s=3 / 2 \otimes 1 / 2=1 \oplus 2$, that is $J^{P}=\left(1^{+}, 2^{+}\right)$, corresponding to $D_{1}$ and $D_{2}^{*}$. The $D_{0}^{*}, D_{1}^{\prime}, D_{1}$ and $D_{2}^{*}$ states are generically denoted as $D^{* *}$.

Other excited discovered states are associated with $2 S$ and $1 D$ wave function of the charmed system. When $n$, the radial excitation quantum number, is larger than 1 , as in the $2 S$ state, they are said to be radially excited.

Parity and angular momentum conservation constrain the decays allowed for each state, helping to experimentally identify the $D^{* *}$ candidates. The $j_{l}=1 / 2$ states decay through a $S$-wave to $D^{(*)} \pi$, and they are both expected to be broad (large decay widths), while the $j_{l}=3 / 2$ states decay to the same states through a $D$-wave and are expected to be more narrow states (small widths).

Recent data coming from $e^{+} e^{-}$colliders identify the four candidates to $D^{* *}$ states with $D_{0}^{*}(2400), D_{1}^{\prime}(2430), D_{1}(2420)$ and $D_{2}^{*}(2460)$. The latest observation of $\bar{B} \rightarrow D^{* *} l \bar{\nu}_{l}$ has been reported by Babar 17 . The rates for the $D^{* *}$ narrow states are in good agreement with the 2005 measurements by $\mathrm{D} \varnothing[18$; the ones for the broad states are in agreement with DELPHI [19], but do not agree with the $D_{1}^{\prime}$ limit of Belle 20. Measurements also indicate that the relation expected in the heavy mass limit 2123

$$
\Gamma\left(B \rightarrow D^{* *}\left(j_{l}=\frac{3}{2}\right) l \bar{\nu}\right) \gg \Gamma\left(B \rightarrow D^{* *}\left(j_{l}=\frac{1}{2}\right) l \bar{\nu}\right)
$$

may be violated; this is known as the " $1 / 2$ vs $3 / 2$ puzzle". Moreover, there has been a long standing problem with the measured semileptonic branching fractions, that can be determined with good precision by integrating the differential decays rates for $\bar{B} \rightarrow D^{(*)} l \bar{\nu}$ decays. The sum of the measured exclusive rates is less than the inclusive one: explicitly

$$
\begin{aligned}
& \mathcal{B}\left(B^{+} \rightarrow X_{c} l^{+} \nu\right)-\mathcal{B}\left(B^{+} \rightarrow D l^{+} \nu\right)-\mathcal{B}\left(B^{+} \rightarrow D^{(*)} l^{+} \nu\right) \\
& -\mathcal{B}\left(B^{+} \rightarrow D^{(*)} \pi l^{+} \nu\right)=(1.45 \pm 0.67) \%
\end{aligned}
$$

with a similar relation holding for the corresponding $B^{0}$ decays 20,24 .

The broadness of $j=1 / 2$ states may be one reason causing the disagreement within experiments and with theory, since it has always been quite difficult to disentangle very broad resonances from continuum, both on theoretical and experimental sides. On these premises, it has been suggested to clarify the comparison between theory and experiment analyzing states analogous to $D_{0}^{*}$ and $D_{1}^{\prime}$, but narrow, in particular studying the decay $B_{s}^{0} \rightarrow \bar{D}_{s J} \pi$ [25. Other theoretical suggestions to ease or solve the previous problems include taking into account an unexpectedly large $B$-decay rate to the first radially excited $D^{\prime(*)}, 13,26$.

In 2010 Babar has found evidence for two new states [27], which may be identified with the $2 S$ states in the quark model picture. Moreover, new data has recently become available on semileptonic $B$-decays to final states containing a $D_{s}^{(*)+} K$ 
system, providing information about the poorly known region of hadronic masses above $2.46 \mathrm{GeV} / \mathrm{c}^{2}$, that covers radially excited $D$-meson states 28. 29 .

\section{Exclusive $\left|V_{u b}\right|$ determination}

The analysis of exclusive charmless semileptonic decays is currently employed to determine the CKM parameter $\left|V_{u b}\right|$. Here we focus on the channel $\bar{B} \rightarrow \pi l \bar{\nu}_{l}$, where recent progress has been reported from both experimental and theoretical sides. Other interesting channels are $B \rightarrow \omega l \nu$ [30] and $B \rightarrow \eta^{\prime} l \nu$ [31, 32]. The $\bar{B} \rightarrow \pi l \bar{\nu}_{l}$ decay is the simplest to interpret, as it is affected by a single form factor $f_{+}\left(q^{2}\right)$ (in the limit of zero leptonic mass)

$$
\frac{d \Gamma\left(\bar{B} \rightarrow \pi l \bar{\nu}_{l}\right)}{d q^{2}}=\frac{G_{F}^{2}\left|p_{\pi}\right|^{3}}{24 \pi^{3}}\left|V_{u b}\right|^{2}\left|f_{+}\left(q^{2}\right)\right|^{2}
$$

where $q$ is the momentum of the leptonic pair and $p_{\pi}$ is the momentum of the pion in the $B$ meson rest frame.

Most studied routes to calculate the form factor are once again lattice and lightcone QCD sum rules. Theoretical predictions for the form factor split into two: predictions for the form factor normalization $f_{+}(0)$ and for the functional form of the $q^{2}$ dependence.

By using current lattice QCD methods, the hadronic amplitudes for $\bar{B} \rightarrow \pi l \bar{\nu}_{l}$ can be calculated quite accurately because there is only a single stable hadron in both the initial and final states. The first results based on unquenched simulations have been obtained by the Fermilab/MILC collaboration [33 and the HPQCD collaboration [34, and they are in substantial agreement. Such high-statistics calculations have been performed in the kinematic region where the outgoing light hadron carries little energy $\left(q^{2} \geq 16 \mathrm{GeV}^{2}\right)$. At low $q^{2}$, with light hadrons carrying large momentum of order $2 \mathrm{GeV}$, direct simulations require a very fine lattice which is not yet accessible in calculations with dynamical fermions. Such fine lattice would also be required to simulate heavy quarks; alternately, one can resort to effective heavy quark theory. It is also helpful to rely on extrapolations from larger momentum transfer $q^{2}$. In Ref. [33], the $b$-quark is simulated by using the so-called Fermilab heavy-quark method, while the dependence of the form factor from $q^{2}$ is parameterized according to the z-expansion 35 37. In Ref. 34, the $b$-quark is simulated by using nonrelativistic QCD and the Becirevic Kaidalov (BK) 38] parameterization is adopted for the $q^{2}$ dependence. Recent results are also available on a fine lattice (lattice spacing $a \sim 0.04 \mathrm{fm}$ ) in the quenched approximations by the QCDSF collaboration [39].

Combining lattice QCD for the $\bar{B} \rightarrow \pi l \bar{\nu}_{l}$ form factor 33,34 with measurements from Belle [40] gives $\left|V_{u b}\right|=(3.43 \pm 0.33) \times 10^{-3}$, while latest Babar data [41 for $\left|V_{u b}\right|$ range from $(3.3-3.5) \times 10^{-3}$. The results are compatible with the value of $\left|V_{u b}\right|$ determined from the $B^{+} \rightarrow \omega l^{+} \nu$ [41], while a value of $\left|V_{u b}\right|$ is not extracted from the $B^{+} \rightarrow \eta l^{+} \nu$ decays because the theoretical partial decay rate is not sufficiently precise for these decays. 
In the sum rule approach, the $B \rightarrow \pi$ matrix element is obtained from the correlation function of quark currents, such that, at large spacelike external momenta, the operator-product expansion (OPE) near the light-cone is applicable. Within OPE, the correlation function is factorized in a series of hard-scattering amplitudes convoluted with the pion light-cone distribution amplitudes of growing twist. Several recent calculations of the semileptonic form factor have become available, based on the light cone QCD sum rules $42[48]$. Direct calculations, without extrapolations, hold in the kinematic region of large recoil, with an upper limit for $q^{2}$ varying between 6 and $16 \mathrm{GeV}^{2}$. The QCD sum rules provide an approximation for the product $f_{B} f_{+}\left(q^{2}\right)$, and therefore the decay constant $f_{B}$ represents a necessary input for the extraction of the form factor. In Ref. [47, and in several other papers, $f_{B}$ is calculated for consistency within the QCD sum rule approach, that is replacing $f_{B}$ by its two-point QCD (SVZ) sum rule 49,50 . In this way one expects that radiative corrections affect in the same way $f_{B}$ and $f_{B} f_{+}\left(q^{2}\right)$, and cancel in the ratio $\left(f_{B} f_{+}\right) / f_{B}$, together with some theoretical uncertainty from input parameters in common, e.g., the $b$ mass. Using the form factor results in Ref. 47] and the latest Babar data [41], the estimate value is $\left|V_{u b}\right|=\left(3.46 \pm 0.06 \pm 0.08_{-0.32}^{+0.37}\right) \times 10^{-3}$ (Ref. 41]), where the three uncertainties are statistical, systematic and theoretical, respectively.

Last year, Babar performed a simultaneous fit to the data over the full $q^{2}$ range and the FNAL/MILC lattice QCD results, publishing the following average value $\left|V_{u b}\right|=(2.95 \pm 0.31) \times 10^{-3}[51$. This year, they have performed a similar fit, with updated data, new lattice results, and some different theoretical assumptions [41]. In Table VII of Ref. [41], for each lattice and QCD sum rule computation, values of $\left|V_{u b}\right|$ are presented, together with the corresponding $\chi^{2} /$ ndf, that can be as low as $\chi^{2} / \mathrm{ndf}=2.7 / 4$ (with probability equal to $60.1 \%$ ) in the HPQCD case. The average estimate [41, determined from the simultaneous fit to experimental data and the lattice theoretical predictions, yields

$$
\left|V_{u b}\right|=(3.25 \pm 0.31) \times 10^{-3}
$$

that is about 1 standard deviation higher than the 2011 estimate. This fairly large difference has been explained by the fact the determination of $\left|V_{u b}\right|$ from the combined data-lattice fit is most sensitive to the points at high $q^{2}$, where the changes due to the improved hybrid treatment have lead to differences larger than those expected on the basis of the variation in the total branching fraction value.

\section{Inclusive semileptonic $B$-decays kinematics}

Let us consider the $\bar{B} \rightarrow X_{q} l \bar{\nu}$ decays, where the final state $X_{q}$ is an hadronic state originated by the quark $q$. In inclusive decays, we sum over all possible final states $X_{q}$, no matter if single-particle or multi-particle states. Since inclusive decays do not depend on the details of final state, quark-hadron duality is generally assumed. We can factorize long distance dynamics of the meson using an OPE approach. 
Another advantage, due to the large mass of the $b$-quark, is the possibility of using the systematic framework provided by the Heavy Quark Effective Theory (HQET). The aftermath of the previous approaches is that inclusive transition rates have the form of a heavy quark expansion, that one can schematically write as

$$
\Gamma\left(B \rightarrow X_{q} l \nu\right)=\frac{G_{F}^{2} m_{b}^{5}}{192 \pi^{3}}\left|V_{q b}\right|^{2}\left[c_{3}<O_{3}>+c_{5} \frac{<O_{5}>}{m_{b}^{2}}+c_{6} \frac{<O_{6}>}{m_{b}^{3}}+O\left(\frac{1}{m_{b}^{4}}\right)\right]
$$

Here $c_{d}$ are short distance coefficients, calculable in perturbation theory as a series in the strong coupling $\alpha_{s}$, and $O_{d}$ denote local operators of (scale) dimension $d$ :

$$
<O_{d}>\equiv \frac{<B\left|O_{d}\right| B>}{2 m_{B}}
$$

where $m_{B}$ is the $B$-meson mass, included in the definition for the normalization and dimensional counting. It is basically the same cast of operators, albeit with different weights, that appears in semileptonic, radiative and nonleptonic rates as well as distributions. The short distance coefficients $c_{d}$ contain the masses of the final state quarks (from phase space, etc.), that require definition in a chosen scheme. The hadronic expectation values of the operators $O_{d}$ encode the nonperturbative corrections. While we can identify these operators and their dimensions, which then determine the power of $1 / m_{b}$, in general we cannot compute their hadronic expectation values from first principles, and we have to rely on a number of HQET parameters, which increases with powers of $1 / m_{b}$. The hadronic expectation value of the leading operator $O_{3}=\bar{b} b$ incorporates the parton model result which dominates asymptotically, i.e. for $m_{b} \rightarrow \infty$. A remarkable feature of Eq. (17) is the absence of a contribution of order $1 / m_{b}$, due to the absence of an independent gauge invariant operator of dimension four. The fact that nonperturbative, bound state effects in inclusive decays are strongly suppressed (at least two powers of the heavy quark mass) explains a posteriori the success of parton model in describing such processes.

The phase space region includes a region of singularity, also called endpoint region, corresponding to a kinematic region near the limits of both the lepton energy $E_{l}$ and $q^{2}$ phase space, where the rate is dominated by the production of low mass final hadronic states. The region of singularity is a cut, since the hadronic final mass can vary, in contrast with exclusive decays. Near the cut, and especially near the endpoints of the cut, the use of the OPE cannot be rigorously justified because there will be propagators that have denominators close to zero. Corrections can be large and need to be resummed at all orders. A resummation formalism analogous to the one used to factorize Sudakov threshold effects for parton distribution functions in usual hard processes, such as deep inelastic scattering or Drell-Yan, can be applied to the case of inclusive heavy meson decays (see e.g. [52 55$]$ ). 


\section{The semi-leptonic $\bar{B} \rightarrow X_{c} l \bar{\nu}$}

Schematically, the decay rate takes the form

$$
\Gamma\left(B \rightarrow X_{c} l \nu\right)=\frac{G_{F}^{2} m_{b}^{5}}{192 \pi^{3}}\left|V_{c b}\right|^{2}\left[f(\rho)+k(\rho) \frac{\mu_{\pi}^{2}}{m_{b}^{2}}+g(\rho) \frac{\mu_{G}^{2}}{m_{b}^{2}}+O\left(\frac{1}{m_{b}^{3}}\right)\right]
$$

where $\rho=m_{c}^{2} / m_{b}^{2}$ and the coefficients $f, k$ and $g$, calculable in perturbation theory, are expressed as a series in $\alpha_{s}$. This expansion is valid only for sufficiently inclusive measurements and away from perturbative singularities, therefore the relevant quantities to be measured are global shape parameters (the first few moments of various kinematic distributions) and the total rate. While the general structure of the expansion is the same for all the above mentioned observables, the perturbative coefficients are in general different.

The leading term is the parton model, which is known completely to order $\alpha_{s}$ and $\alpha_{s}^{2}$, for the width and moments of the lepton energy and hadronic mass distributions 56 59]. The terms of order $\alpha_{s}^{n+1} \beta_{0}^{n}$, where $\beta_{0}$ is the first coefficient of the QCD $\beta$ function, have been included by the usual BLM procedure 60,61 .

For the total rate, the kinetic corrections have the same coefficient as the leading order, $k(\rho)=-f(\rho)$. For other observables, such as partial rates and moments, the kinetic corrections can be obtained from the leading-power differential rate, but the relations are more complicated and only corrections of order $O\left(\alpha_{s} \mu_{\pi}^{2} / m_{b}^{2}\right)$ have been evaluated 62.

For the total rate, the Eq. (19) is known up to order $1 / m_{b}^{5}$, where the terms of order $1 / m_{b}^{n}$ with $n>2$ have been computed only at tree level [63,64]. Up to the higher order available, there are not unnaturally large coefficients, which seem to confirm the duality assumption. At order $1 / m_{b}^{3}$, contributions with an infrared sensitivity to the charm mass $m_{c}$ start to appear. At higher orders, terms such as $1 / m_{b}^{3} m_{c}^{2}$ and $\alpha_{s}\left(m_{c}\right) 1 / m_{b}^{3} m_{c}$ are comparable in size to the contributions of order $1 / m_{b}^{4}$. The total $O\left(1 / m_{Q}^{4,5}\right)$ correction to the width is about $+1.3 \%$, in the approximation that the many HQET non-perturbative parameters are estimated in the ground state saturation approximation 64]. On the same premises, the estimated effect on the $\left|V_{c b}\right|$ determination is a $0.4 \%$ increase.

As it is well known, any quantitative statement about the value of a quark mass must make careful reference to the particular theoretical framework that is used to define it. This scheme dependence also affects the determination of the HQET non-perturbative parameters. Some traditional schemes for masses are not as advantageous in the present context. The pole scheme is most convenient from the point of view of computation, but plagued by large misbehaved higher-order corrections. The minimal subtraction scheme (MS) sets the scale of order of the $b$ quark mass, which is considered unnaturally high, due to the presence of typical scales significantly below, down to the order of $1 \mathrm{GeV}$. Alternative schemes are the so called low subtracted mass schemes, where non perturbative contribution to the heavy quark pole mass can be subtracted by making contact to some physical observable. In recent literature, the latter schemes are commonly used, in particular 
the kinetic 65 66 and 1S scheme 67,68]. Care must be taken in converting from one mass scheme to another due to the presence of truncated perturbative expression.

As mentioned in the previous section, a common feature to many processes in QCD is the presence in the perturbative expansion of large double (Sudakov-like) logarithms at the threshold, in the OPE singularity region. Resummation of large infrared logarithms is essential in order to predict accurate cross sections in many phenomenologically relevant processes. For $b \rightarrow c$ decays, corrections are expected not as singular as in the $b \rightarrow u$ case, because they are cut-off by the charm mass. Nevertheless, their size needs to be estimated and large corrections to be resummed (see e.g. 69,70).

In order to determine $\left|V_{c b}\right|$, a global fit may be performed to the width and all available measurements of moments in $B \rightarrow X_{c} l \nu_{l}$. A global fit has been recently accomplished in both the kinetic and the $1 \mathrm{~S}$ scheme 6. Each scheme has its own non-perturbative parameters that have been estimated together with the charm and bottom masses. The fit constrains only a linear combination of $m_{b}$ and $m_{c}$, not enough to bound the $b$ mass precisely, which reflects on the precision of the determination of $\left|V_{c b}\right|$. This limitation has been overcome by including the photon energy moments in $B \rightarrow X_{s} \gamma$ into the fit, or by applying a precise constraint on the $c$-quark mass. With the former constraint, in the $1 \mathrm{~S}$ scheme, the result of the fit gives

$$
\left|V_{c b}\right|=(41.96 \pm 0.45) \times 10^{-3}
$$

and a very close result

$$
\left|V_{c b}\right|=(41.88 \pm 0.73) \times 10^{-3}
$$

is obtained also in the kinetic scheme, using the $c$-quark mass constraint.

The averages are in agreement with the values given in Sect. 2, extracted from exclusive decays. The most precise measurements are from inclusive, that are below $2 \%$. Still, the determination of $\left|V_{c b}\right|$ from $\bar{B} \rightarrow D^{*} l \bar{\nu}$ has reached the relative precision of about $2 \%$.

We can also compare with the global fit of the CKM matrix elements within the Standard Model, as calculated by the CKMfitter and UTfit groups. The CKMfitter group uses a standard $\chi^{2}$-like statistical frequentist hypothesis, in addition to the RFit scheme for the treatment of theoretical systematics. A recent determination of $\left|V_{c b}\right|$ [71] gives

$$
\left|V_{c b}\right|=(40.69 \pm 0.99) \times 10^{-3}
$$

with pull, that is the difference between measurement and predictions, equal to 0.2. The UTfit uses the Bayesian statistics to extract the observables, and Gaussian parton distribution functions to represent statistical and systematic uncertainties. Their latest estimate gives 72

$$
\left|V_{c b}\right|=(42.3 \pm 0.9) \times 10^{-3}
$$

with pull less than 1 . All estimates agree within the errors. 


\subsection{Inclusive $V_{u b}$ determination}

The smallest element in the CKM mixing matrix $\left|V_{u b}\right|$ plays a crucial role in the study of the unitarity constraints and of the related fundamental questions. In principle, the method of extraction of $\left|V_{u b}\right|$ from inclusive $\bar{B} \rightarrow X_{u} l \bar{\nu}_{l}$ decays follows in the footsteps of the $\left|V_{c b}\right|$ determination. However, experimentally, the copious background from the $\bar{B} \rightarrow X_{c} l \bar{\nu}_{l}$ process, which has a rate about 50 times higher, does not makes feasible a measurement over the full phase space. To overcome this background, inclusive $\bar{B} \rightarrow X_{u} l \bar{\nu}_{l}$ measurements utilize restricted regions of phase space in which the $\bar{B} \rightarrow X_{c} l \bar{\nu}_{l}$ process is highly suppressed by kinematics. This requires knowledge of the fraction of the total $\bar{B} \rightarrow X_{u} l \bar{\nu}_{l}$ that lies within the utilized section of phase space, which complicates the theoretical issues considerably. The region where the background is forbidden overlaps with the end-point, or singularity, region, where the energy and mass of the hadron final state are of order $E_{X} \simeq m_{b}$ and $m_{X}^{2} \sim \Lambda_{Q C D} m_{b} \ll m_{b}^{2}$, respectively. This kinematic region has sufficient phase space for many different resonances to be produced in the final state, so an inclusive description of the decays is still appropriate. However, here, the differential rate is very sensitive to the details of the wave function of the $b$ quark in the $B$-meson. The nonperturbative effects related to a small vibration of the heavy quark in the $B$-meson (the so-called Fermi motion) are enhanced. The parton level differential distribution at the end-point region has its own problems, as well, related to the presence of soft and collinear singularities. Large logarithms appear, which spoil the perturbative expansion and need to be resummed at all orders. A way to remedy the previous difficulties is to introduce a non-perturbative form factor, the shape function (see, e.g., Refs. 7379 ). It can be interpreted, from a physical point of view, as the distribution of the effective mass of the heavy quark at disintegration time. At leading order in $\Lambda_{Q C D} / m_{b}$, the shape function can be extracted from a reference process, such as the radiative $B \rightarrow X_{s} \gamma$, and used to predict other inclusive $B$-meson decays. At higher orders, the shape function is no more universal, its functional form is unknown and one has to resort to model functions to interpret the measured differential distributions.

The discrepancy between the values of $\left|V_{u b}\right|$ extracted from inclusive and exclusive decays is a long standing problem, and a lot of effort has already been devoted to find possible solutions. Two main routes to progress in the extraction of $\left|V_{u b}\right|$ can be identified. The first one is to enlarge the experimental range, so as to reduce, on the whole, the weight of the endpoint region. Latest results by Belle 80 access $\sim 90 \%$ of the $\bar{B} \rightarrow X_{u} l \bar{\nu}_{l}$ phase space, claiming an overall uncertainty of $7 \%$ on $\left|V_{u b}\right|$. A similar portion of the phase space is covered also by the most recent Babar analysis 81 . The second route is to enlarge our theoretical prospective, by comparing results obtained in several available theoretical schemes. All of them are tailored to analyze data in the threshold region, but differ significantly in their treatment of perturbative corrections and the parameterization of non-perturbative effects.

The latest experimental determinations of $\left|V_{u b}\right|$ come from Babar 81] and HFAG 
6] collaborations. They both extract $\left|V_{u b}\right|$ from the partial branching fractions relying on at least four different QCD calculations of the partial decay rate: BLNP by Bosch, Lange, Neubert, and Paz 82 84; DGE, the dressed gluon exponentiation, by Andersen and Gardi 85; ADFR by Aglietti, Di Lodovico, Ferrara, and Ricciardi 86 88]; and GGOU by Gambino, Giordano, Ossola and Uraltsev [89]. A comparison of the above approaches generally leads to roughly consistent results when the same inputs are used and the theoretical errors are taken into account. In Fig 1 we plot the values for $\left|V_{u b}\right|$ (without the errors) and each point correspond to a different experiment (sensible to a different experimental cut) used as an input in the HFAG average [6]. The listed experiments and cuts are respectively, from left to right: CLEO, where the cut imposed on the leptonic energy has been $2.1<E_{e}<2.6$ (Ref. [90]); Belle, where the analysis has been performed with limits on both the invariant mass $\left(m_{X}<1.7 \mathrm{GeV}\right)$ and the lepton pair squared momentum $\left(q^{2}>8 \mathrm{GeV}^{2}\right)$ (Ref. [91]) or with $1.9<E_{e}<2.6$ (Ref. 92]); Babar, with a constraint $2.0<E_{e}<2.6$ (Ref. 93]); Belle, with a cut on the lepton energy at $1 \mathrm{GeV}$ (Ref. [80]); the remaining points refer to Babar, where various cuts have been imposed, as can be seen in Ref. 81 .

\section{$\left|v_{u b}\right|\left(10^{-3}\right)$}

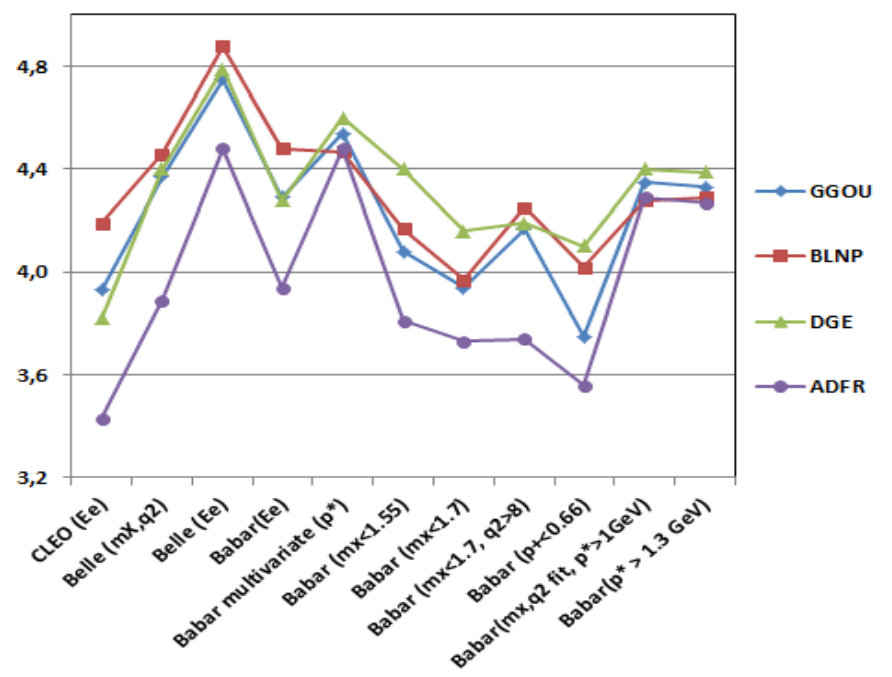

Fig. 1. Comparison of different $\left|V_{u b}\right|$ values (without the errors) obtained in different theoretical approaches. Blu, red, green and violet lines refer, respectively, to GGOU, BLNP, DGE, ADFR. Each point correspond to a different experiment (listed in the text).

Other proposed theoretical approaches 94,96 have not been included in Fig 1 since they do not provide the same extensive list of $\left|V_{u b}\right|$ determination for each experiment as the previous approaches. The latest method advanced [97] aims at 
providing a global fit to the available data in inclusive $B \rightarrow X_{s} \gamma$ and $B \rightarrow X_{u} l \nu_{l}$ decays. No estimate of $\left|V_{u b}\right|$ within this approach is available at the moment.

Notwithstanding the proliferation of theoretical methods and approaches, the values of $\left|V_{u b}\right|$ extracted from inclusive decays maintain about two $\sigma$ above the values given by exclusive determinations. Also indirect fits prefer a lower value of $\left|V_{u b}\right|$. Very recent CKMfitter results give 71

$$
V_{u b}=\left(3.42_{-0.1}^{+0.2}\right) \times 10^{-3}
$$

For Utfit 72

$$
V_{u b}=(3.62 \pm 0.14) \times 10^{-3}
$$

In Fig. 2 we plot a summary table of the inclusive averages from Ref. [6] and compare with indirect results, Eq. 24) and Eq. 25), and Babar latest exclusive fit, Eq. 16. The closest value to the exclusive determination comes from the ADFR approach and reads

$$
V_{u b}=\left(4.03 \pm 0.13_{-0.12}^{+0.18}\right) \times 10^{-3}
$$

where the errors quoted correspond to experimental and theoretical uncertainties, respectively.

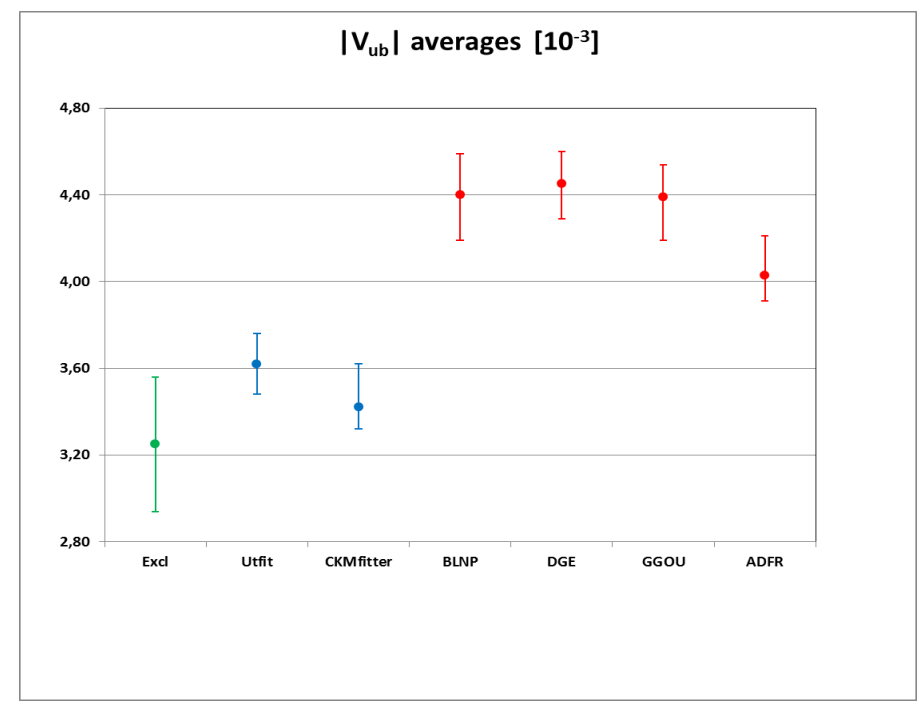

Fig. 2. Comparison of $\left|V_{u b}\right|$ values extracted from exclusive, inclusive decays and indirect fits. 


\section{2. $B_{s}^{0}$ decays}

$B_{s}$ decays are attracting a lot of attention, due to the avalanche of recent data and to the expectation of new ones. Apart from standard form factors and branching ratio computations, the inclusive $B_{s}$ decays can be usefully compared with the inclusive $B$-decays to check quark-hadron duality and evaluate heavy quark expansion parameters, while exclusive decays represent an interesting probe to analyze the (expected sizable) $S U(3)$ breaking.

There are well known differences between the $B_{s}^{0}$ and the $B^{0}$ system. The mixing parameter $x_{s} \equiv \Delta m_{s} / \Gamma_{s}$ is about 30 times larger than $x_{d}$, and the mass and width difference are sizable. Another important difference is that the $\mathrm{CP}$ violating mixing phase probes the angle $\beta_{s}$ in the unitarity triangle, which is about two order of magnitudes smaller than $\beta$ in the Standard Model, and hence negligibly small. Any large variation due to new physics can produce observable effects, and that alone would be enough to motivate the study of $\mathrm{CP}$ violation in the $B_{s}^{0}$ system. For a very recent brief review on $\mathrm{CP}$ violation in the $B_{s}^{0}$ system see, e.g., Ref. 98 .

In 2010 a discrepancy with the standard model has been reported in the measurement of the (like-sign) dimuon charge asymmetry $\mathcal{A}_{s l}^{b}$ of semi-leptonic decays of $b$ hadrons. The anomalous $\mathcal{A}_{s l}^{b}$, found by the experiment $\mathrm{D} \varnothing$ with $6 \mathrm{fb}^{-1}$ of data, deviated $3.2 \sigma$ from the SM 99 . The $2011 \mathrm{D} \varnothing$ update at $9 \mathrm{fb}^{-1}$ shows again a deviation, at $3.9 \sigma 100$, from the Standard Model value 101

$$
\begin{aligned}
\mathcal{A}_{s l}^{b} & =\left[-0.787 \pm 0.172_{(\text {stat })} \pm 0.093_{(\text {syst })}\right] \% \\
\mathcal{A}_{s l}^{b}(\mathrm{SM}) & =\left[-0.028_{-0.006}^{+0.005}\right] \%
\end{aligned}
$$

$\mathcal{A}_{s l}^{b}$ is defined as the difference in the number of events with a pair of positive muons minus the number with a pair of negative muons divided by the sum.

A related observable is the semileptonic charge asymmetry $a_{s l}$, defined as

$$
a_{s l}=\frac{\Gamma\left(\bar{B}_{s}^{0}(t) \rightarrow f\right)-\Gamma\left(B_{s}^{0}(t) \rightarrow \bar{f}\right)}{\Gamma\left(\bar{B}_{s}^{0}(t) \rightarrow f\right)+\Gamma\left(B_{s}^{0}(t) \rightarrow \bar{f}\right)}=\frac{1-|q / p|^{4}}{1+|q / p|^{4}}
$$

testing the "wrong" final state, accessible only through mixing. The asymmetry $a_{s l}$ measures $\mathrm{CP}$ violation in mixing and it is independent from time and from the final state (to within a sign), as it can always be ascribed to a property of the decaying states. At lowest order in $\left|\Gamma_{12} / M_{12}\right|$, we have

$$
\left|\frac{q}{p}\right|^{2}=1-a \quad a \equiv \operatorname{Im}\left(\frac{\Gamma_{12}}{M_{12}}\right)=\frac{\Delta \Gamma_{s}}{\Delta m_{s}} \tan \phi_{s}
$$

where $\phi_{s} \equiv \arg \left(-M_{12} / \Gamma_{12}\right), \Delta m_{s} \equiv m_{H}-m_{L}=2\left|M_{12}\right|$ and $\Delta \Gamma_{s}=\Gamma_{L}-\Gamma_{H}=$ $2\left|\Gamma_{12}\right| \cos \phi_{s}$. Notice that the symbol $\phi_{s}$ is overloaded, since in literature it is used also for the $\mathrm{CP}$ violating phases defined in a slight different way. Whatever the definition, the phase can be related to $\beta_{s}$, that in the Standard Model is $\beta_{s} \equiv$ $\arg \left[-V_{t b}^{*} V_{t s} / V_{c b}^{*} V_{c s}\right]$, since the dispersive term of the weak hamiltonian $M_{12}$ is mainly driven by box diagrams involving virtual top quarks and the absorptive term $\Gamma_{12}$ is dominated by on-shell charmed intermediate states. An addition of a 
non-standard phase, e. g. $\beta_{s}(S M) \rightarrow \beta_{s}(S M)+\tilde{\beta}_{s}$, it is often used to parameterize effects of new physics or non-leading hadronic contributions.

Since it arises from the meson mixing, if there is not a separation of the asymmetry due to $B^{0}$ and $B_{s}^{0}, \mathcal{A}_{s l}^{b}$ can be written as

$$
\mathcal{A}_{s l}^{b}=C_{d} a_{s l}^{d}+C_{s} a_{s l}^{s}
$$

where the coefficients depend on mean mixing probability and the production rates of $B^{0}$ and $B_{s}^{0}$ mesons. Here $a_{s l}^{d}$ is the semileptonic charge asymmetry in the $B^{0}$ system, which has been measured since 2001 at $e^{+} e^{-}$machines. The actual averaged value from CLEO, Babar, Belle, Opal and Aleph collaborations is $a_{s l}^{d}=(-0.10 \pm$ $0.37) \%$ 6].

The value of $a_{s l}^{s}$, semileptonic charge asymmetry in the $B_{s}^{0}$ system, extracted from the $\mathrm{D} \varnothing$ measurement 100 reads

$$
a_{s l}^{s}=(-1.81 \pm 1.06) \%
$$

The semileptonic charge asymmetry $a_{s l}^{s}$ has also been directly measured by the experiment $\mathrm{D} \varnothing$ via the decay $B_{s}^{0} \rightarrow D_{s}^{-} \mu^{+} X$, using data corresponding to $5 \mathrm{fb}^{-1}$ of integrated luminosity $a_{s l}^{s}=(-0.17 \pm 0.91 \text { (stat) })_{-0.23}^{+0.12}$ (syst) $) \% 102$. A new and improved measurement of $a_{s l}^{s}$ using the full Tevatron data sample with an integrated luminosity of $10.4 \mathrm{fb}^{-1}$ gives 103

$$
a_{s l}^{s}=\left(-1.08 \pm 0.72_{(\text {stat })} \pm 0.17_{(\text {syst })}\right) \%
$$

The value of the Standard Model prediction for $a_{s l}=(1.9 \pm 0.3) \times 10^{-5}[101]$ is negligible compared with current experimental precision. The extracted value for $a_{s l}^{s}$ is in agreement with the direct and the Standard Model determinations. First LHC-b results are already available, from measurements of $B^{0} \rightarrow D^{ \pm} \mu^{\mp} \nu$ and $B_{s}^{0} \rightarrow D_{s}^{ \pm} \mu^{\mp} \nu$ asymmetries, giving 104

$$
a_{s l}^{s}=\left(-0.24 \pm 0.54_{(\text {stat })} \pm 0.33_{(\text {syst })}\right) \%
$$

Recent precise determinations of the CP asymmetry in several non-leptonic decays already severely constrain a possible interpretation of the like-sign dimuon charge asymmetry in terms of non-standard CP-violating contributions to $B_{s}$ mixing, while more precision in traditional and new channels is expected soon (see, e.g., Refs. 105 107]). Greatly improved precision or, even better, independent measurements of semileptonic asymmetries are needed to establish evidence of $\mathrm{CP}$ violation due to new physics in semi-leptonic $B_{s}^{0}$ decays.

\section{References}

1. A. J. Buras, Lectures given at the 38th Course of the Erice International School of Subnuclear Physics: Theory and Experiment Heading for New Physics, 27/8-5/9, 2000, hep-ph/0101336.

2. G. Ricciardi, arXiv:1209.3386 [hep-ph].

3. M. Okamoto et al., Nucl. Phys. Proc. Suppl. 140, 461 (2005). 
4. B. Aubert et al. [BABAR Collaboration], Phys. Rev. D 79, 012002 (2009).

5. B. Aubert et al. [BABAR Collaboration], Phys. Rev. Lett. 104, 011802 (2010).

6. Y. Amhis et al. [Heavy Flavor Averaging Group Collaboration], arXiv:1207.1158 [hepex].

7. G. M. de Divitiis et al., Phys. Lett. B 655, 45 (2007).

8. G. M. de Divitiis, R. Petronzio and N. Tantalo, JHEP 0710, 062 (2007).

9. C. Bernard et al. [Fermilab Lattice and MILC Collaborations], Phys. Rev. D 79, 014506 (2009).

10. J. A. Bailey et al. [Fermilab Lattice and MILC Collaboration], PoS LATTICE 2010, $311(2010)$.

11. C. Bernard et al. [Fermilab Lattice and MILC Collaborations], Phys. Rev. D 78, 094505 (2008).

12. P. Gambino, T. Mannel and N. Uraltsev, Phys. Rev. D 81, 113002 (2010).

13. P. Gambino, T. Mannel and N. Uraltsev, arXiv:1206.2296 [hep-ph]

14. A. S. Kronfeld, Phys. Rev. D 62, 014505 (2000).

15. N. Uraltsev, Phys. Lett. B 585, 253 (2004).

16. J. Beringer et al. [Particle Data Group Collaboration], Phys. Rev. D 86, 010001 (2012).

17. B. Aubert et al. [BABAR Collaboration], Phys. Rev. Lett. 101, 261802 (2008).

18. V. Abazov et al. [DØ Collaboration], Phys. Rev. Lett. 95, 171803 (2005).

19. J. Abdallah et al. [DELPHI Collaboration], Eur. Phys. J. C 4535 (2006).

20. D. Liventsev et al. [Belle Collaboration], Phys. Rev. D 77, 091503 (2008).

21. A. K. Leibovich, Z. Ligeti, I. W. Stewart and M. B. Wise, Phys. Rev. D 57, 308 (1998).

22. N. Uraltsev, Phys. Lett. B 501, 86 (2001).

23. I. I. Bigi et al., Eur. Phys. J. C 52, 975 (2007).

24. B. Aubert et al. [BABAR Collaboration], Phys. Rev. Lett. 100, 151802 (2008).

25. D. Becirevic et al., arXiv:1206.5869 [hep-ph].

26. F. U. Bernlochner, Z. Ligeti, S. Turczyk, Phys.Rev. D85, 094033 (2012).

27. P. del Amo Sanchez et al. [BABAR Collaboration], Phys. Rev. D82, 111101 (2010)

28. P. del Amo Sanchez et al. [BABAR Collaboration], Phys. Rev. Lett. 107, 041804 (2011)

29. J. Stypula et al. [Belle Collaboration], arXiv:1207.6244 [hep-ex].

30. J. P. Lees et al. (BABAR Collaboration), arXiv:1205.6245v1.

31. P. del Amo Sanchez et al. [BABAR Collaboration], Phys. Rev. D 83, 052011 (2011).

32. C. Di Donato, G. Ricciardi and I. Bigi, Phys. Rev. D 85013016 (2012).

33. J. A. Bailey et al., Phys. Rev. D 79, 054507 (2009).

34. E. Dalgic, A. Gray, M. Wingate, C. T. H. Davies, G. P. Lepage and J. Shigemitsu, Phys. Rev. D 73, 074502 (2006) [Erratum-ibid. D 75, 119906 (2007)].

35. T. Becher and R. J. Hill, Phys. Lett. B 633, 61 (2006).

36. M. C. Arnesen et al., Phys. Rev. Lett. 95, 071802 (2005).

37. C. Bourrely, I. Caprini, and L. Lellouch, Phys. Rev. D 79, 013008 (2009).

38. D. Becirevic and A. B. Kaidalov, Phys. Lett. B 478, 417 (2000).

39. A. Al-Haydari et al. [QCDSF Collaboration], Eur. Phys. J. A 43, 107 (2010) arXiv:0903.1664 [hep-lat]].

40. H. Ha et al. [Belle Collaboration], Phys. Rev. D 83071101 (2011).

41. J. P. Lees et al. [BABAR Collaboration], arXiv:1208.1253 [hep-ex].

42. P. Ball and R. Zwicky, J. High Energy Phys. 10, 019 (2001).

43. P. Ball and R. Zwicky, Phys. Rev. D 71, 014015 (2005)

44. P. Ball and G. W. Jones, JHEP 08, 025 (2007).

45. A. Khodjamirian et al., Phys. Rev. D 62, 114002 (2000).

46. G. Duplancic et al., J. High Energy Phys. 04, 014 (2008). 
47. A. Khodjamirian et al., Phys. Rev. D 83, 094031 (2011).

48. A. Bharucha, JHEP 1205, 092 (2012).

49. M.A. Shifman, A.I. Vainshtein, and V.I. Zakharov, Nucl. Phys. B 147, 385 (1979).

50. M.A. Shifman, A.I. Vainshtein, and V.I. Zakharov, Nucl. Phys. B 147, 448 (1979).

51. P. del Amo Sanchez et al. [BABAR Collab.], Phys. Rev. D 83032007 (2011)

52. U. Aglietti and G. Ricciardi, Phys. Rev. D 66, 074003 (2002).

53. U. Aglietti, G. Ricciardi and G. Ferrera, Phys. Rev. D 74, 034004 (2006).

54. U. Aglietti, G. Ricciardi and G. Ferrera, Phys. Rev. D 74, 034005 (2006).

55. U. Aglietti, G. Ricciardi and G. Ferrera, Phys. Rev. D 74, 034006 (2006).

56. M. Trott, Phys. Rev. D 70, 073003 (2004).

57. A. Pak and A. Czarnecki, Phys. Rev. D 78, 114015 (2008).

58. S. Biswas and K. Melnikov, JHEP 1002, 089 (2010).

59. P. Gambino, JHEP 1109, 055 (2011).

60. D. Benson et al., Nucl. Phys. B 665, 367 (2003).

61. V. Aquila, P. Gambino, G. Ridolfi and N. Uraltsev, Nucl. Phys. B 719, 77 (2005).

62. T. Becher, H. Boos, and E. Lunghi, JHEP 0712, 062 (2007).

63. I. I. Bigi, N. Uraltsev, and R. Zwicky, Eur. Phys. J.C 50, 539 (2007).

64. T. Mannel, S. Turczyk and N. Uraltsev, JHEP 1011, 109 (2010).

65. I.I.Y. Bigi et al., Phys. Rev. D 52, 196 (1995).

66. D. Benson, I. I. Bigi, T. Mannel, and N. Uraltsev, Nucl. Phys. B 665, 367 (2003),

67. A.H. Hoang et al., Phys. Rev. D 59, 074017 (1999).

68. C. W. Bauer, Z. Ligeti, M. Luke, A. V. Manohar, and M. Trott, Phys. Rev. D 70, 094017 (2004).

69. U. Aglietti, L. Di Giustino, G. Ferrera, A. Renzaglia, G. Ricciardi and L. Trentadue, Phys. Lett. B 653, 38 (2007).

70. L. Di Giustino, G. Ricciardi and L. Trentadue, Phys. Rev. D 84, 034017 (2011).

71. D. Derkach, talk given at Implications of $\mathrm{LHCb}$ measurements and future prospects workshop, CERN, Geneva, April 16-18, 2012

72. C. Tarantino, talk given at ICHEP2012, Melbourne, July 4-12, 2012

73. I. Bigi, M. Shifman, N. Uraltsev and A. Vainshtein, Phys. Rev. Lett. 71, 496 (1993).

74. A. Manohar and M. Wise, Phys. Rev. D 49, 1310 (1994).

75. M. Neubert, Phys. Rev. D 49, 3392 (1994), ibid. 4623 (1994).

76. I. Bigi, M. Shifman, N. Uraltsev and A. Vainshtein, Int. J. Mod. Phys. A 9, 2467 (1994)

77. T. Mannel and M. Neubert, Phys. Rev. D 50, 2037 (1994).

78. U. Aglietti and G. Ricciardi, Phys. Lett. B 466, 313 (1999).

79. U. Aglietti and G. Ricciardi, Nucl. Phys. B 587, 363 (2000).

80. P. Urquijo et al. [Belle Collaboration], Phys. Rev. Lett. 104, 021801 (2010).

81. J. P. Lees et al. [BABAR Collaboration], Phys. Rev. D 86, 032004 (2012).

82. B. O. Lange, M. Neubert, and G. Paz, Phys. Rev. D 72, 073006 (2005).

83. S.W. Bosch, B. O. Lange, M. Neubert, and G. Paz, Nucl. Phys. B 699, 335 (2004).

84. S. W. Bosch, M. Neubert, and G. Paz, JHEP 11, 073 (2004).

85. J. R. Andersen and E. Gardi, JHEP 0601, 097 (2006),

86. U. Aglietti and G. Ricciardi, Phys. Rev. D 70, 114008 (2004).

87. U. Aglietti, G. Ferrera and G. Ricciardi, Nucl. Phys. B 768, 85 (2007).

88. U. Aglietti, F. Di Lodovico, G. Ferrera and G. Ricciardi, Eur. Phys. J. C 59, 831 (2009)

89. P. Gambino, P. Giordano, G. Ossola, and N. Uraltsev, JHEP 0710, 058 (2007).

90. A. Bornheim et al. [CLEO Collaboration], Phys. Rev. Lett. 88, 231803 (2002)

91. H. Kakuno et al. [BELLE Collaboration], Phys. Rev. Lett. 92, 101801 (2004). 
92. A. Limosani et al. [Belle Collaboration], Phys. Lett. B 621, 28 (2005).

93. B. Aubert et al. [BABAR Collaboration], Phys. Rev. D 73, 012006 (2006)

94. C. W. Bauer, Z. Ligeti and M. E. Luke, Phys. Rev. D 64, 113004 (2001).

95. A. K. Leibovich, I. Low and I. Z. Rothstein, Phys. Rev. D 61, 053006 (2000).

96. B. O. Lange, M. Neubert and G. Paz, JHEP 0510, 084 (2005).

97. Z. Ligeti, I. W. Stewart, and F. J. Tackmann, Phys. Rev. D 78, 114014 (2008).

98. G. Ricciardi, in Proceedings of Rencontres de Moriond, QCD and High Energy Interactions, La Thuile, March 10-17, 2012, arXiv:1205.2752 [hep-ph].

99. V. M. Abazov et al. [DØ Collaboration], Phys. Rev. Lett. 105, 081801 (2010).

100. V. M. Abazov et al. [DØ Collaboration], Phys. Rev. D 84, 052007 (2011).

101. A. Lenz and U. Nierste, J. High Energy Phys. 06, 072 (2007).

102. V. M. Abazov et al. [DØ Collaboration], Phys. Rev. D 82, 012003 (2010) [Erratumibid. D 83119901 (2011)].

103. V. M. Abazov et al. [D0 Collaboration], arXiv:1207.1769 [hep-ex].

104. The LHCb collaboration, LHCb-CONF-2012-022, Conference report prepared for the 36th ICHEP, Melbourne, July 2012.

105. R. Aaij et al. [LHCb Collaboration], Phys. Rev. Lett. 108, 101803 (2012).

106. R. Fleischer, R. Knegjens and G. Ricciardi, Eur. Phys. J. C 71, 1798 (2011).

107. R. Fleischer, R. Knegjens and G. Ricciardi, Eur. Phys. J. C 71, 1832 (2011). 